\title{
Audit of CT scanning of paranasal sinuses in patients referred with facial pain*
}

Ida Amir, Justin C.L Yeo, Bhaskar Ram

Department of Otolaryngology and Head and Neck Surgery, Aberdeen Royal Infirmary, Aberdeen, Scotland, United Kingdom
Rhinology 50: 442-446, 2012

DOI:10.4193/Rhino.12.036

*Received for publication:

February 25, 2012

Accepted: June 27, 2012

\section{SUMMARY}

Background: Computed tomography (CT) scans are performed by some clinicians in the belief that they are a useful primary investigation in patients with facial pain.

Objective: To assess the appropriateness and outcome of sinuses CT scans in patients with facial pain based on the European Position Paper on Chronic Rhinosinusitis and Nasal Polyps (EPOS) 2007 guideline and International Headache Society (IHS) criteria for diagnosing and investigating rhinosinusitis.

Methodology: The first cycle of audit was performed on 50 patients with facial pain who underwent CT scanning. The findings on nasal endoscopy, Lund-Mackay scores (LMS) of the scans and management of these patients were analysed. Following implementation of the IHS and EPOS criteria, 50 consecutive patients were re-audited.

Results: In the first cycle, $16 \%$ of patients had positive nasal endoscopic findings. Thirty patients had LMS of 0 and only 9 showed significant changes ( $L M S \geq 8$ ) on their scans. In the second cycle, only 10 patients underwent CT imaging as per EPOS guideline and 4 of them showed significant changes. The remaining $80 \%$ of patients in this cycle were diagnosed and treated for non-sinogenic causes.

Conclusion: Applying the IHS and EPOS criteria has reduced the number of inappropriate CT scans requests and allowed consideration of non-sinogenic aetiologies.

Key words: facial pain, headache, sinusitis, CT scan

\section{Introduction}

Facial pain is a common complaint in the otolaryngology outpatient setting and has numerous aetiologies for clinicians to consider. A comprehensive account of these causes can be found in the International Headache Society (IHS) Classification, which includes both neurological and non-neurological aetiologies ${ }^{(1)}$. In the evaluation of facial pain, the primary goal for the otolaryngologist is to make a distinction between sinogenic and non-sinogenic causes. The complexity in managing facial pain is also often contributed by patients' pre-conception that their symptoms are attributed to their sinuses.

Accurate history taking is essential in obtaining the correct diagnosis in patients with facial pain. The classic approach is to focus on the structures from which pain arises. Due to the similarities in pain localisation from non-sinogenic causes, there is a tendency to diagnose facial pain that is associated with rhinological symptoms as chronic rhinosinusitis (CRS). This usually lead clini- 
cians to request computed tomography (CT) scans of paranasal sinuses as a primary investigation ${ }^{(2)}$. The International Headache Society (IHS) does not validate chronic rhinosinusitis as a cause of headache or facial pain unless relapsing into an acute stage ${ }^{(1)}$. Furthermore, the European Position Paper on Chronic Rhinosinusitis and Nasal Polyps (EPOS) 2007 stated that the diagnosis of CRS in patients with facial pain or pressure should be accompanied by two or more symptoms, one of which should be either nasal blockage/obstruction/congestion or nasal discharge (anterior/posterior nasal drip). Although CT scanning is the imaging modality of choice confirming the extent of pathology and the anatomy for example in surgical planning, it should not be used as the primary investigation except where there are unilateral or sinister signs and symptoms, or after failure of medical therapy (3).

This audit was performed to assess the appropriateness and outcome of CT scans of paranasal sinuses in patients referred with facial pain in the National Health Service (NHS) Grampian catchment area based on the standards outlined by the IHS and EPOS 2007; and whether applying these standards will avoid unnecessary CT scanning.

\section{Materials and methods}

\section{Standards for audit}

The diagnostic criteria for headache attributed to rhinosinusitis according to IHS are ${ }^{(1)}$ :

Frontal headache accompanied by pain in one or more regions of the face, ears or teeth and fulfilling criteria $C$ and $D$;

Clinical, nasal endoscopic, CT and/or MRI imaging and /or laboratory evidence of acute or acute-on-chronic rhinosinusitis; Headache and facial pain that developed simultaneously with onset or acute exacerbation of rhinosinusitis;

Headache and/or facial pain resolved within days after remission or successful treatment of acute or acute-on-chronic rhinosinusitis.

The EPOS 2007 diagnostic criteria fo $r$ CRS are summarised below ${ }^{(3)}$ : Inflammation of the nose and the paranasal sinuses characterised by two or more symptoms, one of which should be either:

- Nasal blockage/obstruction/congestion or nasal discharge (anterior or posterior drip)

- $\quad+$ - facial pain/pressure;

- $\quad$ /- reduction or loss of smell;

- for more than 12 weeks;

accompanied by endoscopic signs of:

- Polyps and/ or mucopurulent discharge primarily from middle meatus

- and/ or oedema or mucosal obstruction primarily in middle meatus
CT scan is not recommended in both acute and chronic rhinosinusitis unless additional problems such as:

- $\quad$ very severe disease;

- immunocompromised patients;

- $\quad$ signs of complications.

CT scan can be useful in corroborating history and examination after failure of medical therapy or to demonstrate sinonasal anatomy for pre-operative planning.

\section{Clinical data}

The first cycle of audit was performed as a retrospective case notes review of new patients who were referred to the ENT clinic by their primary care practitioner for facial pain and subsequently, had CT scan of the paranasal sinuses. Fifty patients who underwent the CT scanning from January 2010 to June 2010 were selected. Data collected from the case notes of these patients include the indication for the scan, presence of rhinological or other symptoms, nasal endoscopy and other clinical examination findings. The CT scans were analysed and presence of CRS were staged using the Lund-Mackay scoring (LMS) system. The outcome after the CT scan was also analysed in terms of final diagnosis and further management.

Following the implementation of the diagnostic criteria from both IHS and EPOS, our clinical practice was re-audited. This was conducted by analysing 50 consecutive new outpatient facial pain referrals from primary care in NHS Grampian to the local ENT department. These patients suffered from chronic facial pain and did not have prior ENT examination or CT scan. The referral letters for these patients stated a request for ENT input in further management of their chronic rhinosinusitis conditions. A prospectively kept database held in the secure hospital network was designed with the aim of capturing data on patients' facial pain symptoms, diagnosis as well as management plan. The results from both stages were compared and analysed.

This audit was approved by the NHS Grampian Clinical Effectiveness Unit and was assigned project ID 2145.

\section{Results}

The first part of our audit showed that 37 of 50 patients with facial pain had at least one of rhinological symptoms (nasal obstruction, rhinorrhoea, post-nasal drip and/or anosmia). Eight patients had positive nasal endoscopy findings of CRS, which showed pus or mucosal disease at the middle meatus or presence of nasal polyps. Twenty-one patients had completely normal nasal endoscopic findings. The rest of 21 patients had septal deviation and or inferior turbinate hypertrophy. Further details of endoscopic findings are shown in Table 1.

Clinical details provided in the CT scan requests were also exa- 
Table 1. Characteristics of the first and re-audit cycles data.

First cycle

Total no. of patients

Presence of rhinological symptom/s

\section{0}

37

$\begin{array}{cc}50 & 50 \\ 21 & 31 \\ 8 & 10 \\ 17 & 7 \\ 4 & 2\end{array}$

50

\section{1}

Nasal endoscopy examination

Normal

Evidence of CRS

Septal deviation with or without inferior turbinate hypertrophy

Inferior turbinate hypertrophy only

\begin{tabular}{lll}
\hline Outcome after CT scan/further management & 9 & 4 \\
\hline Endoscopic sinus surgery & 8 & 0 \\
\hline Septoplasty +/- reduction of inferior turbinates & 25 & 6 \\
\hline Medical treatment for CRS & 8 & 40 \\
Medical treatment for non-sinogenic facial pain & 0 & 6 \\
Mid-facial segment pain & 3 & 3 \\
Atypical pain & 5 & 15 \\
Migraine & 0 & 2 \\
\hline Temporomandibular joint dysfunction & 0 & 5 \\
Cluster headache/Trigeminal autonomic cephalagias & 0 & 3 \\
Tension Type Headache & 0 & 6
\end{tabular}

Re-audit cycle

200

$\begin{array}{ll}50 & 10 \\ 30 & 2 \\ 11 & 4 \\ 0 & 0 \\ 9 & 4\end{array}$

mined. Only 2 requests stated the indication for the scan were for pre-operative planning. The rest of 48 requests indicated that the scan were to assist in diagnosing CRS. Thirty-six \% of patients complained of pain over maxillary sinus, $16 \%$ over the frontal sinus and $14 \%$ in the periorbital region. The other $34 \%$ of patients had pain at other sites, which include temporal, teeth, retro-orbital and pre-auricular. Thirty patients had no evidence of CRS on the scans (LMS $=0$ ) and 11 patients had minimal disease (LMS = 1-4). Nine patients were found to have significant disease on the CT scan (LMS $\geq 8$ ) and all these patients proceeded to have sinus surgery. Eight patients had septoplasty with or without reductions of inferior turbinates. Twenty-five patients continued their medical treatment for CRS. Non-sinogenic cau- ses of facial pain were diagnosed in the remaining 8 patients.

In the second cycle of the audit, 31 out of 50 patients with facial pain analysed had at least one of associated rhinological symptoms. Forty were found not to have evidence of pus, polyp or mucosal disease on nasal endoscopic examination and therefore CT scans were not requested in these patients. Thirty-four of these 40 patients were diagnosed and treated for non-sinogenic causes of facial pain. Six of them did not meet the EPOS criteria for CRS and therefore were diagnosed as non-classifiable facial pain at the initial clinic consultation. Only 10 patients had positive nasal endoscopic findings and proceeded to have CT scan of paranasal sinuses. Four patients had significant muco- 
periosteal disease on the CT scan (LMS $>8$ ) and proceeded to have sinus surgery. The remaining 6 patients (LMS $<4$ ) were treated for non-sinogenic facial pain. The list of diagnosis of non-sinogenic causes of facial pain made in this cycle of study is outlined in Table 1.

\section{Discussion}

Facial pain and headache in the anatomical distribution of the paranasal sinuses pose a clinical challenge. This is usually made complicated when patients also present with rhinological symptoms such as rhinorrhoea, post-nasal drip and nasal obstruction and therefore are often presumed to have CRS. Multiple studies have shown that an average of more than $70 \%$ of patients who meet the diagnostic criteria for migraine are self-diagnosed or diagnosed by their primary care doctors as suffering from 'sinus headache' ${ }^{(4-7)}$. This common misconception is shared not only by many patients but also by their health care professionals. These patients usually had medical treatment for CRS already instigated by their primary care practitioner or through self-medication with little improvement to their facial pain ${ }^{(4)}$. Their referrals often lead to clinicians requesting CT scan of paranasal sinuses to delineate the extent of mucoperiosteal disease.

The EPOS 2007 publication provides clear guidelines on the diagnosis and management of CRS. The diagnosis of CRS is symptom based and CT scans or plain x-rays are not recommended to diagnose this condition. The addition of nasal endoscopy has been reported to improve diagnostic accuracy and should be emphasized as an early diagnostic tool ${ }^{(3)}$. Recent publication of EPOS 2012 placed more emphasis on nasal endoscopy especially in moderate to severe disease ${ }^{(8)}$. Agius found that patients with facial pain as their principal symptoms of CRS were significantly less likely to score positive for CRS on CT scans and those with CT positive were significantly more likely to have evidence of disease on nasal endoscopy ${ }^{\left({ }^{9}\right.}$. The use of nasal endoscopy for investigating facial pain patients with presumed CRS would help to reduce the use of CT scan, costs and radiation exposure.

The International Classification of Headache Disorders stated that chronic rhinosinusitis is not validated as a cause of headache or facial pain unless relapsing into an acute stage ${ }^{(1)}$. Nonsinogenic facial pain such as migraine, tension headache, midfacial segment pain and trigeminal autonomic cephalgias (TAC) may manifest in the anatomical distribution of paranasal sinuses ${ }^{(10)}$. Because of the overlap of distribution and the nature of referral of pain associated with non-sinogenic facial pain disorders, localisation to paranasal sinuses is seldom pathognomic of CRS. We found in this audit that the areas commonly involved are the maxillary and frontal regions, which made up 52\% of anatomical site of pain presentation in our patients in the first part of the study. However, only $16 \%$ of these patients had evidence of CRS on nasal endoscopy.

Autonomic symptoms of lacrimation, nasal congestion and rhinorrhoea may further cause diagnostic confusion in those presenting with facial pain. Patients with migraine or trigeminal autonomic cephalalgia (TAC) may also present with nasal congestion, rhinorrhoea and post-nasal drips ${ }^{(10-12)}$. TAC is a group of primary headache disorders which include cluster headache, paroxysmal hemicrania, short lasting unilateral neuralgiform headache attacks with conjunctival injection and tearing (SUNCT), and short-lasting unilateral neuralgiform headache attacks with cranial autonomic symptoms (SUNA) ${ }^{(1)}$. In 1908, Sluder described a group of neuralgic, motor, sensory and gustatory symptoms and signs. He hypothesised that these clinical findings were due to inflammation of the sphenopalatine ganglion and advocated topical treatment of the ganglion using cocaine or formaldehyde ${ }^{(13)}$. The condition has now been classified under cluster headache due to common autonomic features seen with vascular type headaches. Schreiber et al., reported that on average of $60 \%$ of patients with IHS-defined migraine reported nasal congestion and runny nose, and $84 \%$ of them reported sinus pain ${ }^{(4)}$. In both cycles of this study, we found that on average of $68 \%$ of patients with facial pain also complained of at least one rhinological symptom. In these patients, only $18 \%$ of them had positive nasal endoscopic findings of CRS, which showed pus, polyp or mucosal oedema at the middle meatus.

In the evaluation of patients with facial pain, history taking and physical examination with nasal endoscopy are essential in differentiating between sinogenic and non-sinogenic causes. A study of 108 patients who had mucopurulent discharge at endoscopy found that only $29 \%$ of them complained of facial pain ${ }^{(12)}$. The diagnosis of CRS can be also made by applying the EPOS diagnostic criteria. EPOS only recommends the use of CT scan in complicated cases, those that failed maximum medical therapy and to delineate anatomy in surgical planning ${ }^{(3)}$. Many studies support this recommendation as CT scanning in CRS has been shown to have poor correlation with patients' symptoms and more than a third of patients may have incidental findings $(12,14,15)$.

We found that in the first cycle of our audit, CT scans were inappropriately performed on patients presented with facial pain. We acknowledge that it is difficult to ascertain in this study whether these patients had optimum medical treatment for their presumed CRS and therefore had CT scans requested for further investigation or pre-operative planning. We could only assume the indications for these patients based on the details provided on the request forms. Therefore, we can conclude that in the first cycle of this audit that the CT scans were inappropria- 
tely requested in $84 \%$ of patients. In the second cycle, we found that application of IHS definition of CRS and EPOS guideline in diagnosing, investigating and managing CRS are not only helpful in preventing unnecessary CT scanning but also in the workup of non-sinogenic causes. However, CT or magnetic resonance imaging (MRI) should be performed in patients presenting with warning signs or red flag features of potential secondary causes (16). Imaging should also be considered if the underlying disease or condition cannot be diagnosed with thorough history and clinical examination and or if patients not responding to medical treatments.

Due to the complex nature and various aetiologies associated with facial pain, we also advocate a multidisciplinary team (MDT) approach in order to provide a more comprehensive assessment and management to these patients. Our facial pain MDT at the NHS Grampian currently comprises various clinical specialties which include the otolaryngology, neurology, neurosurgery, oral and maxillofacial; and anaesthesiology. This set up allows a more pragmatic approach to patient care and avoid extensive and unnecessary investigation.

\section{Conclusion}

The CT scan of paranasal sinuses is a necessary tool for preoperative planning. Application of criteria for diagnosis and management of CRS avoid unnecessary scanning in facial pain and allow for consideration of other potential diagnosis of non-sinogenic causes. Our audit also indicates that rhinological symptoms are common in facial pain and their presence should not signify a diagnosis of CRS. Imaging modalities are useful in complex cases especially in those with potential secondary causes.

\section{Authors' contribution}

IA performed literature search and review, and wrote this manuscript. JY and BR assisted in writing and editing this manuscript. All authors were involved in data collection and analysis.

\section{Conflict of interest}

No conflict of interests are declared.

\section{References}

1. Headache Classification Committee of the International Headache Society. International Classification of Headache Disorders (2nd Edition). Cephalalgia. 2004; 24: 114-121.

2. Stewart MG, Johnson RF. Chronic sinusitis: symptoms versus CT scan findings. Curr Opin Otolaryngol Head Neck Surg. 2004; 12: 27-29.

3. Fokkens W, Lund V, Mullol J. European Position Paper on Rhinosinusitis and Nasal Polyps group. European position paper on rhinosinusitis and nasal polyps 2007. Rhinol Suppl. 2007; 45: 1-139.

4. Schreiber CP, Hutchinson S, Webster CJ, Ames M, Richardson MS, Powers C. Prevalence of migraine in patients with a history of self-reported or physician-diagnosed "sinus" headache. Arch Intern Med. 2004; 164: 1769-1772.

5. Lipton RB, Diamond S, Reed M, Diamond $M L$, Stewart WF. Migraine diagnosis and treatment: results from the American Migraine Study II. Headache. 2001; 41: 638645.

6. Tepper SJ. New thoughts on sinus headache. Allergy Asthma Proc. 2004; 25: 95-96.
7. Eross E, Dodick D, Eross M. The sinus, allergy and migraine study (SMAS). Headache. 2007; 47: 213-227.

8. Fokkens W, Lund V, Mullol J, et al. EPOS 2012: European position paper on rhinosinusitis and nasal polyps 2012. A summary for otorhinolaryngologists. Rhinology. 2012; 50: 1-12.

9. Agius A. M. Long term follow-up of patients with facial pain in chronic rhinosinusitis correlation with nasal endoscopy and CT. Rhinology. 2010; 48: 65-70.

10. Barbanti P, Fabbrini G, Perare M. Unilateral cranial autonomic symptoms in migraine. Cephalalgia. 2002; 22: 256-259.

11. Edvinsson L. Aspects on the pathophysiology of migraine and cluster headache. Pharmacol Toxicol. 2001; 89: 65-73.

12. Clifton N, Jones NS. The prevalence of facial pain in 108 consecutive patients with paranasal mucopurulent discharge at endoscopy. J Laryngol Otol. 2007; 121: 345-348.

13. Sluder $\mathrm{G}$. The role of the sphenopalatine (or Meckel's) ganglion in nasal headaches. New York Medical Journal. 1908; 87: 989-990.

14. Marshall A, Jones NS. The utility of radiological studies in the diagnosis and management of rhinosinusitis. Curr Infect Dis Rep.
2003; 5: 199-204.

15. Shields G, Seikaly H, LeBoeuf M, Guinto F, LeBoeuf H, Pincus T, Calhoun K. Correlation between facial pain or headache and computed tomography in rhinosinusitis in Canadian and U.S. subjects. Laryngoscope. 2003; 113: 943-945.

16. Scottish Intercollegiate Guidelines Network. Diagnosis and management of headache in adults. A national clinical guideline. Edinburgh. SIGN; 2008.

Miss Ida Amir

Specialty Registrar

Department of Otolaryngology and

Head and Neck Surgery

Aberdeen Royal Infirmary

Scotland

United Kingdom

E-mail: i.amir@nhs.net 